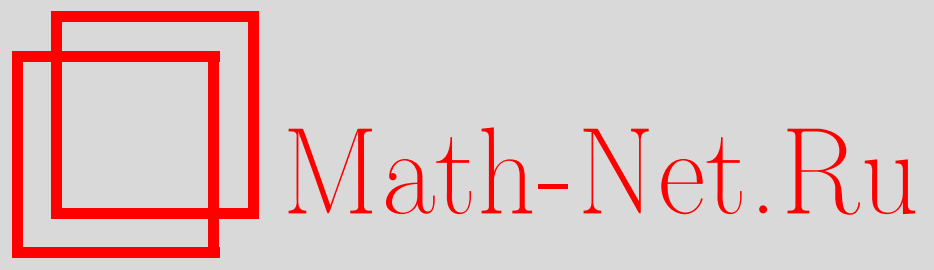

А. Л. Рухин, Корреляционные матрицы цепочек для марковских последовательностей и тестирование случайности, Теория вероятн. и ее примен., 2006, том 51, выпуск 4, 712-731

DOI: https://doi.org/10.4213/tvp21

Использование Общероссийского математического портала MathNet.Ru подразумевает, что вы прочитали и согласны с пользовательским соглашением

http: //www . mathnet.ru/rus/agreement

Параметры загрузки:

IP: 54.237 .206 .68

26 апреля 2023 г., 15:27:04

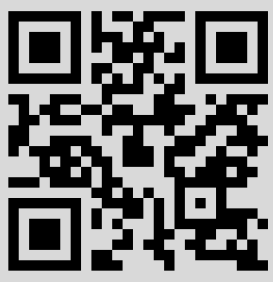




\title{
КОРРЕЛЯЦИОННЫЕ МАТРИЦЫ ЦЕПОЧЕК ДЛЯ МАРКОВСКИХ ПОСЛЕДОВАТЕЛЬНОСТЕЙ И ТЕСТИРОВАНИЕ СЛУЧАЙНОСТИ
}

\begin{abstract}
В статье установлены свойства корреляционных матриц цепочек, которые полезны для статистических выводов о марковских последовательностях. Получены асимптотические разложения для. вероятности появления заданного слова предписанное число раз, а также для совместного появления двух слов. Эти разложения дают хорошие приближения для двух первых моментов частот появлений. Найдено выражение для ковариационной матрицы совместного распределения этих частот через корреляционную матрицу цепочек и получен вид псевдообратной для такой ковариационной матрицы. Указываются статистические применения для проверки случайности методом хи-квадрат.
\end{abstract}

Ключевые слова и фразы: асимптотические разложения, резольвента, производящая функция вероятностей, псевдообратная матрица, хи-квадрат, фундаментальная матрица.

1. Введение. Рассматривается случайная последовательность букв из конечного алфавита. Задача состоит в определении вероятности появления некоторой цепочки заданное число раз. Эта задача возникает в теории информации и в молекулярной биологии. Обзор [14] содержит конкретные биологические применения. В предположении независимости букв формула для упомянутой вероятности выражается через корреляционный полином цепочек, введенный в [6], или через матрицу, образованную такими полиномами [19].

В настоящей статье корреляционная матрица цепочек определяется для эргодической сложной цепи Маркова. Получена система уравнений, связывающая эту матрицу и производящую функцию вероятностей появления цепочек из данного множества. Найдены асимптотические разложения для вероятности появления заданной цепочки определенное число раз, а также вероятности совместного появления двух цепочек. Эти разложения ведут к удивительно точным приближенным формулам

* Department of Mathematics and Statistics, UMBC, Baltimore, MD 21250 USA and Statistical Engineering Division, National Institute of Standards and Technology, Gaithersburg, MD 20899 USA; e-mail: rukhin@math.umbc.edu 
для моментов случайного числа появления этих цепочек. Выводятся формулы для математического ожидания числа цепочек, появляющихся заданное число раз, и выясняется ковариационная структура соответствующих случайных величин. Как следствие дается вид оптимального линейного теста случайности для близких альтернатив. В качестве примера рассмотрен случай 2-грамм в марковской цепи порядка два. Наконец, ковариационная матрица совместных частот выражена через корреляционную матрицу. Получена ее псевдообратная и указаны применения к проверке данных вероятностей букв.

2. Корреляционные матрицы цепочек. Рассмотрим последовательность случайных величин $\varepsilon_{k}, k=1, \ldots, n$, принимающих значения в $\mathscr{I}=\{1, \ldots, q\}$ при некотором $q \geqslant 2$. Предполагается, что эта последовательность образует цепь Маркова порядка не больше $m$.

Зачастую изучение таких последовательностей основывается на свойствах налагающихся $m$-цепочек $\left(m\right.$-грамм) $\left(i_{1} \ldots i_{m}\right)$ при фиксированном $m$. Обозначим через $\mathscr{I}_{m}$ множество всех таких цепочек, общее число которых есть $q^{m}$.

Предположим, что для $\imath=\left(i_{1} \ldots i_{m}\right)$ все вероятности $P(\imath)=$ $P\left\{\left(\varepsilon_{1}, \ldots, \varepsilon_{m}\right)=\left(i_{1} \ldots i_{m}\right)\right\}$ положительны. Пусть для $k=1, \ldots, m$

$$
\begin{aligned}
P\left(j_{m-k+1} \ldots j_{m} \mid \imath\right)=P\{ & \left(\varepsilon_{m+1}, \ldots, \varepsilon_{m+k}\right)=\left(j_{m-k+1} \ldots j_{m}\right) \\
& \left.\mid\left(\varepsilon_{1}, \ldots, \varepsilon_{m}\right)=\left(i_{1} \ldots i_{m}\right)\right\}=\frac{P\left(\imath j_{m-k+1} \ldots j_{m}\right)}{P(\imath)}
\end{aligned}
$$

означает условную вероятность того, что цепочке $j_{k+1} \ldots j_{m}$ предшествует $\imath$.

Корреляиионный полином цепочек $\widetilde{C}_{\imath \jmath}(z)$ был введен в [6]. Пусть $\imath=\left(i_{1} \ldots i_{m}\right)$ и $\jmath=\left(j_{1} \ldots j_{m}\right)$ - две цепочки из $\mathscr{I}_{m}$. Положим

$$
\begin{aligned}
\widetilde{C}_{\imath \jmath}(z) & =\sum_{r=0}^{m-1} \delta_{\left(i_{r+1} \ldots i_{m}\right),\left(j_{1} \ldots j_{m-r}\right)} P\left(j_{m-r+1} \ldots j_{m} \mid \imath\right) z^{r} \\
& =\delta_{\imath, \jmath}+\sum_{r=1}^{m-1} \delta_{\left(i_{r+1} \cdots i_{m}\right),\left(j_{1} \ldots j_{m-r}\right)} P\left(j_{m-r+1} \ldots j_{m} \mid \imath\right) z^{r} .
\end{aligned}
$$

Здесь и далее $\delta_{\imath, \jmath}=\prod_{k=1}^{m} \delta_{i_{k} j_{k}}$ - символ Кронекера для двух $m$-грамм $\imath$ и л. Если $\varepsilon_{k}$ независимы и равномерно распределены, определение в [6] относится к многочлену $z^{m-1} \widetilde{C}_{\imath \jmath}(q / z)$. Наше же определение следует работе [15], где при $\imath=\jmath$ изучается случай обычных марковских цепей.

При заданном $\imath$ обозначим через $P^{(r)}(\jmath \mid \imath)$ условную вероятность того, что цепочка л следует за $\imath$ после $r$ букв,

$$
P^{(r)}(\jmath \mid \imath)=\sum_{k_{1}, \ldots, k_{r}} P\left(k_{1} \ldots k_{r} \jmath \mid \imath\right),
$$


а при $0 \leqslant z<1$ положим

$$
\mathscr{C}_{\imath \jmath}(z)=\widetilde{C}_{\imath \jmath}(z)+\sum_{r=0}^{\infty} z^{m+r}\left[P^{(r)}(\jmath \mid \imath)-P(\jmath)\right] .
$$

Коррелячионная матрица чепочек $\mathscr{C}(z)=\mathscr{C}$ размера $q^{m} \times q^{m}$ состоит из элементов $\mathscr{C}_{\imath \jmath}(z), \imath, \jmath \in \mathscr{I}_{m}$. Она использовалась в [19] для последовательностей Бернулли, когда $P^{(r)}(\jmath \mid \imath)=P(\jmath)$, так что $\mathscr{C}_{\imath \jmath}=\widetilde{C}_{\imath \jmath}$. Автор в [17] применил эту матрицу для нахождения производящих функций вероятностей появления заданных частот.

Обозначим через I единичную матрицу, размер которой обычно будет ясен, через $\mathbf{p}$ вектор из единиц размерности $q^{m}$; е будет обозначать вектор размерности $q^{m}$ с координатами $P(\imath), \imath \in \mathscr{I}_{m}$. Элементы матрицы $\mathbf{P}$ определены как

$$
P_{\imath \jmath}=\delta_{\left(i_{2} \ldots i_{m}\right),\left(j_{1} \ldots j_{m-1}\right)} P\left(\varepsilon_{n+m}=j_{m} \mid \varepsilon_{n}=i_{1}, \ldots, \varepsilon_{n+m-1}=i_{m}\right) .
$$

Элементы матриц $\mathbf{P}^{k}$ при $k=1, \ldots, m-1$ имеют вид

$$
\delta_{\left(i_{k+1} \ldots i_{m}\right),\left(j_{1} \ldots j_{m-k}\right)} P\left(j_{m-k+1} \cdots j_{m} \mid \imath\right) .
$$

Полагая $\mathbf{P}^{0}=\mathbf{I}_{q^{m}}$, видим, что матрица

$$
\widetilde{C}(z)=\sum_{k=0}^{m-1} z^{k} \mathbf{P}^{k}
$$

образована корреляционными полиномами.

Так как

$$
\begin{aligned}
& P\left(j_{m-r-1} \ldots j_{m-1} \mid i_{m} k_{1} \ldots k_{r} k_{r+1} j_{1} \ldots j_{m-r-2}\right) P\left(j_{m} \mid k_{r+1} j_{1} \ldots j_{m-1}\right) \\
& =P\left(j_{m-r-1} \ldots j_{m-1} \mid i_{m} k_{1} \ldots k_{r} k_{r+1} j_{1} \ldots j_{m-r-2}\right) \\
& \quad \times P\left(j_{m} \mid i_{m} k_{1} \ldots k_{r} k_{r+1} j_{1} \ldots j_{m-1}\right) \\
& =P\left(j_{m-r-1} \ldots j_{m} \mid i_{m} k_{1} \ldots k_{r} j_{1} \ldots j_{m-r-1}\right),
\end{aligned}
$$

по индукции получаем элементы $\mathbf{P}^{m+r}, r=0,1,2, \ldots$ :

$$
\begin{aligned}
& \sum_{k_{1}, \ldots, k_{r}} P\left(k_{1} \ldots k_{r} j_{1} \ldots j_{m-r-1} \mid \imath\right) P\left(j_{m-r} \ldots j_{m} \mid i_{m} k_{1} \ldots k_{r} j_{1} \ldots j_{m-r-1}\right) \\
& \quad=\sum_{k_{1}, \ldots, k_{r}} P\left(k_{1} \ldots k_{r} \jmath \mid \imath\right)=P^{(r)}(\jmath \mid \imath)
\end{aligned}
$$

При $0 \leqslant z<1$ получаем

$$
\begin{aligned}
\mathscr{C}(z) & =\widetilde{C}(z)+\sum_{k=m}^{\infty} z^{k}\left[\mathbf{P}-\mathbf{e p}^{T}\right]^{k}=\widetilde{C}(z)+\sum_{k=m}^{\infty} z^{k}\left[\mathbf{P}^{k}-\mathbf{e p}^{T}\right] \\
& =\sum_{k=0}^{\infty} z^{k} \mathbf{P}^{k}-\frac{z^{m}}{1-z} \mathbf{e p}^{T}=[\mathbf{I}-z \mathbf{P}]^{-1}-\frac{z^{m}}{1-z} \mathbf{e p}^{T}
\end{aligned}
$$


Таким образом, корреляционная матрица цепочек может быть определена посредством (6). Известно, что резольвента $[\lambda \mathbf{I}-\mathbf{P}]^{-1}=\lambda^{-q^{m}}[\mathbf{I}-$ $\left.\lambda^{-1} \mathbf{P}\right]^{-1}$ задана при всех $\lambda$ за исключением собственных значений матрицы $\mathbf{P}(\lambda=1$ - одно из них). Тем самым $\mathscr{C}(z)$ может быть определена при всех комплексных $z$ таких, что $z^{-1}$ не принадлежит спектру $\mathbf{P}$. Так как $\mathbf{P}^{T} \mathbf{p}=\mathbf{p}$ и $\mathbf{P e}=\mathbf{e}$, то следующее определение $\mathscr{C}(z)$ включает случай $z=1$ :

$$
\mathscr{C}(z)=\left[\mathbf{I}-z\left(\mathbf{P}-\mathbf{e p}^{T}\right)\right]^{-1}+\left(z+\cdots+z^{m-1}\right) \mathbf{e p}^{T}
$$

В частности,

$$
\mathscr{C}(1)=\left[\mathbf{I}-\mathbf{P}+\mathbf{e p}^{T}\right]^{-1}+(m-1) \mathbf{e p}^{T} .
$$

Итак, для всех $z$ таких, что $z^{-1}$ не является собственным значением $\mathbf{P}$, имеем

$$
\mathscr{C}_{\imath \jmath}(z)+\frac{z^{m} P(\jmath)}{1-z}=[\mathbf{I}-z \mathbf{P}]_{\imath \jmath}^{-1}=\frac{A_{\jmath \imath}(z)}{\operatorname{det}(\mathbf{I}-z \mathbf{P})},
$$

где $A_{\imath \jmath}(z)$ обозначает алгебраическое дополнение элемента $\imath, \jmath$ в матрице $\mathbf{I}-z \mathbf{P}$. Ясно, что все элементы корреляционной матрицы цепочек $\mathscr{C}(z)$, так же как и матрицы $\mathscr{C}(z)+z^{m} \mathbf{e p}^{T} /(1-z)$ - рациональные функции от $z$. Отметим, что в случае независимых одинаково распределенных величин $\mathbf{P}^{m}=\mathbf{P}^{m+r}=\mathbf{e p}^{T}$, так что $\mathscr{C}(z)=\widetilde{C}(z)$. В этой ситуации все собственные значения $\mathbf{P}$ равны либо 1 (с кратностью один), либо 0 (с кратностью $\left.q^{m}-1\right)$. Тогда $\mathscr{C}(z)$ определена при всех $z$.

Когда $\varepsilon_{1}, \ldots, \varepsilon_{n}$ связаны марковской зависимостью порядка меньше, чем $m$, элементы корреляционной матрицы упрощаются.

Теорема 2.1. Предположим, что случайнье величины $\varepsilon_{1}, \ldots, \varepsilon_{n}$ образуют обычную иепь Маркова с матрицей переходньх вероятностей $\Pi, \Pi_{i j}=P(j \mid i), i, j=1, \ldots, q$. Тогда для $\mathbf{P}$, определенной в (3),

$$
\operatorname{det}\left(\mathbf{I}_{q^{m}}-z \mathbf{P}\right)=\operatorname{det}\left(\mathbf{I}_{q}-z \Pi\right)=(1-z) \Pi\left(1-z \lambda_{k}\right),
$$

где $\lambda_{k}, 0 \leqslant\left|\lambda_{k}\right|<1$ - собственные значения матрицы $\Pi$, отличные от единицьь. Для $0 \leqslant|z|<1$

$$
\begin{aligned}
\mathscr{C}_{\imath \jmath}(z)+\frac{z^{m} P(\jmath)}{1-z}= & \delta_{\imath \jmath}+\sum_{r=1}^{m-1} \delta_{\left(i_{r+1} \ldots i_{m}\right),\left(j_{1} \ldots j_{m-r}\right)} z^{r} \Pi_{j_{m-r} j_{m-r+1}} \cdots \Pi_{j_{m-1} j_{m}} \\
& +z^{m} \Pi_{j_{1} j_{2}} \cdots \Pi_{j_{m-1} j_{m}}\left[\Pi\left(\mathbf{I}-z\left(\Pi-\mathbf{e} \pi^{T}\right)\right)^{-1}\right]_{i_{m} j_{1}} .
\end{aligned}
$$

Если $\pi$ обозначает вектор стационарных вероятностей, то

$$
\begin{aligned}
\mathscr{C}_{\imath \jmath}(z)= & \delta_{\imath \jmath}+\sum_{r=1}^{m-1} \delta_{\left(i_{r+1} \ldots i_{m}\right),\left(j_{1} \ldots j_{m-r}\right)} z^{r} \Pi_{j_{m-r} j_{m-r+1}} \cdots \Pi_{j_{m-1} j_{m}} \\
& +z^{m} \prod_{j_{1} j_{2}} \cdots \prod_{j_{m-1} j_{m}}\left[\Pi\left(\mathbf{I}-z\left(\Pi-\mathbf{e} \pi^{T}\right)\right)^{-1}\right]_{i_{m} j_{1}}-z^{m} P(\jmath) .
\end{aligned}
$$


Д о к а за т е л с т в о. Прежде всего отметим, что

$$
\mathbf{P}=\mathscr{Q}_{1} \mathscr{R}_{1}
$$

где $\mathscr{Q}_{1}$ - матрица размера $q^{m} \times q^{m-1}$, образованная элементами $\delta_{\left(i_{2} \ldots i_{m}\right),\left(j_{1} \ldots j_{m-1}\right)}$, и $\mathscr{R}_{1}$ - матрица размера $q^{m-1} \times q^{m}$, образованная элементами $\delta_{\left(i_{1} \ldots i_{m-1}\right),\left(j_{1} \ldots j_{m-1}\right)} P\left(j_{m} \mid j_{1} \ldots j_{m-1}\right)$. Поскольку ненулевые собственные значения матриц $\mathscr{Q}_{1} \mathscr{R}_{1}$ и $\mathscr{R}_{1} \mathscr{Q}_{1}$ совпадают, имеем

$$
\operatorname{det}\left(\mathbf{I}_{q^{m}}-z \mathbf{P}\right)=\operatorname{det}\left(\mathbf{I}_{q^{m-1}}-z \mathscr{R}_{1} \mathscr{Q}_{1}\right)
$$

Элементы матрицы $\mathscr{R}_{1} \mathscr{Q}_{1}$ суть $\delta_{\left(i_{2} \ldots i_{m-1}\right),\left(j_{1} \ldots j_{m-2}\right)} P\left(j_{m-1} \mid i_{1} \ldots i_{m-1}\right)$. Для марковской цепи порядка $m-2$ эти элементы равны $\delta_{\left(i_{2} \ldots i_{m-1}\right),\left(j_{1} \ldots j_{m-2}\right)} \times$ $P\left(j_{m-1} \mid j_{1} \ldots j_{m-2}\right)$. Таким образом, $\mathscr{R}_{1} \mathscr{Q}_{1}$ совпадает с матрицей $\mathbf{P}$, построенной для $(m-1)$-грамм, и $\mathscr{R}_{1} \mathscr{Q}_{1}=\mathscr{Q}_{2} \mathscr{R}_{2}$ при соответствуюшем определении $\mathscr{Q}_{2}$ как матрицы размера $q^{m-1} \times q^{m-2}$ с элементами $\delta_{\left(i_{3} \ldots i_{m}\right),\left(j_{1} \ldots j_{m-2}\right)}$ и $\mathscr{R}_{2}$ как матрицы размера $q^{m-2} \times q^{m-1}$ с элементами $\delta_{\left(i_{1} \ldots i_{m-2}\right),\left(j_{1} \ldots j_{m-2}\right)} P\left(j_{m-1} \mid j_{1} \ldots j_{m-2}\right)$. Поэтому

$$
\operatorname{det}\left(\mathbf{I}_{q^{m}}-z \mathbf{P}\right)=\operatorname{det}\left(\mathbf{I}_{q^{m-2}}-z \mathscr{R}_{2} \mathscr{Q}_{2}\right) .
$$

По индукции получаем, что

$$
\operatorname{det}\left(\mathbf{I}_{q^{m}}-z \mathbf{P}\right)=\operatorname{det}\left(\mathbf{I}_{q}-z \mathscr{R}_{m-1} \mathscr{Q}_{m-1}\right)
$$

где матрица $\mathscr{R}_{m-1}$ размера $q \times q^{2}$ образована элементами $\delta_{i_{1} j_{1}} P\left(j_{2} \mid j_{1}\right)$, а матрица $\mathscr{Q}_{m-1}$ размера $q^{2} \times q$ имеет элементы вида $\delta_{i_{m} j_{1}}$. Так как $\mathscr{R}_{m-1} \mathscr{Q}_{m-1}=\Pi$, то формула (9) доказана.

Чтобы доказать (10), заметим, что для марковских цепей порядка один

$$
\begin{aligned}
P^{(r)}(\jmath \mid \imath) & =\sum_{k_{1}, \ldots, k_{r}} P\left(k_{1} \ldots k_{r} \jmath \mid i_{m}\right) \\
& =\sum_{k_{1}, \ldots, k_{r}} \Pi_{i_{m} k_{1}} \Pi_{k_{1} k_{2}} \cdots \Pi_{k_{r-1} k_{r}} \Pi_{k_{r} j_{1}} \cdots \Pi_{j_{m-1} j_{m}} \\
& =\Pi_{i_{m} j_{1}}^{(r+1)} \Pi_{j_{1} j_{2}} \cdots \Pi_{j_{m-1} j_{m}}
\end{aligned}
$$

где $\Pi_{i j}^{(r+1)}$ обозначают элементы матрицы $\Pi^{r+1}$. При $0 \leqslant z<1$ получаем

$$
\begin{aligned}
\mathscr{C}_{\imath \jmath}(z)+\frac{z^{m} P(\jmath)}{1-z} & =\widetilde{C}_{\imath \jmath}(z)+\sum_{k=m}^{\infty} z^{k} P^{(k)}(\jmath \mid \imath) \\
& =\widetilde{C}_{\imath \jmath}(z)+\Pi_{j_{1} j_{2}} \cdots \Pi_{j_{m-1} j_{m}} \sum_{k=m}^{\infty} z^{k} \Pi_{i_{m} j_{1}}^{(k+1)} \\
& =\widetilde{C}_{\imath \jmath}(z)+z^{m} \Pi_{j_{1} j_{2}} \cdots \Pi_{j_{m-1} j_{m}}\left[\Pi\left(\mathbf{I}_{q}-z \Pi\right)^{-1}\right]_{i_{m} j_{1}}
\end{aligned}
$$


что равносильно (10). Формула (11) следует отсюда, так как $P(\jmath)=$ $\pi\left(j_{1}\right) \Pi_{j_{1} j_{2}} \cdots \Pi_{j_{m-1} j_{m}}$ и

$$
[\Pi(\mathbf{I}-z \Pi)]^{-1}=\left[\Pi\left(\mathbf{I}-z\left(\Pi-\mathbf{e} \pi^{T}\right)\right)\right]^{-1}+\frac{z}{1-z} \mathbf{e p}^{T}
$$

Теорема 2.1 доказана.

Из доказательства теоремы получается также выражение корреляционной матрицы для марковских цепей порядка меньше, чем $m$, через матрицу переходных вероятностей. При $\imath=\jmath$ соотношение (10) было получено в [15] совершенно другим методом.

Введем теперь симметричную $q^{m} \times q^{m}$-матрицу $\mathbf{V}$ с элементами

$$
\delta_{\left(i_{2} \ldots i_{m}\right),\left(j_{2} \ldots j_{m}\right)}\left[P\left(i_{2} \ldots i_{m}\right)\right]^{-1}=\delta_{\left(i_{2} \ldots i_{m}\right),\left(j_{2} \ldots j_{m}\right)}\left[P\left(j_{2} \ldots j_{m}\right)\right]^{-1}
$$

Легко видеть, что

$$
\mathbf{V p}=\mathbf{e}
$$

Пусть диагональная матрица D образована вектором p. Элементы произведения VD имеют вид

$$
\delta_{\left(i_{2} \ldots i_{m}\right),\left(j_{2} \ldots j_{m}\right)} \frac{P\left(j_{1} \ldots j_{m}\right)}{P\left(j_{2} \ldots j_{m}\right)} .
$$

$\mathrm{B}$ частности, $\operatorname{tr}(\mathbf{V D})=q^{m-1}$, а $\mathbf{V D e}=\mathbf{e . ~ К р о м е ~ т о г о , ~}$

$$
\mathbf{V D V}=\mathbf{V}
$$

Тем самым, обе матрицы DV и VD идемпотентны, и обе имеют ранг $q^{m-1}$.

При условии, что $\varepsilon_{1}, \ldots, \varepsilon_{n}$ связаны марковской зависимостью порядка $m-1$, элементы матрицы VDP имеют вид

$$
\begin{aligned}
& \sum_{k_{1} \ldots k_{m}} \delta_{\left(i_{2} \ldots i_{m}\right),\left(k_{2} \ldots k_{m}\right)} \delta_{\left(k_{2} \ldots k_{m}\right),\left(j_{1} \ldots j_{m-1}\right)} \frac{P\left(k_{1} \ldots k_{m}\right)}{P\left(k_{1} \ldots k_{m-1}\right)} P\left(j_{m} \mid k_{1} \ldots k_{m}\right) \\
& \quad=\sum_{k_{1} \ldots k_{m}} \delta_{\left(i_{2} \ldots i_{m}\right),\left(k_{2} \ldots k_{m}\right)} \delta_{\left(k_{2} \ldots k_{m}\right),\left(j_{1} \ldots j_{m-1}\right)} \frac{P\left(k_{1} \ldots k_{m}\right)}{P\left(k_{1} \ldots k_{m-1}\right)} P\left(j_{m} \mid k_{2} \ldots k_{m}\right) \\
& \quad=\delta_{\left(i_{2} \ldots i_{m}\right),\left(j_{1} \ldots j_{m-1}\right)} P\left(j_{m} \mid i_{2} \ldots i_{m}\right)=\delta_{\left(i_{2} \ldots i_{m}\right),\left(j_{1} \ldots j_{m-1}\right)} P\left(j_{m} \mid i_{1} \ldots i_{m}\right)
\end{aligned}
$$

Тем самым,

$$
\mathbf{V D P}=\mathbf{P}
$$

Формула (13) означает, что при всех $z$, для которых определено $\mathscr{C}(z)$,

$$
\mathbf{V D}[\mathscr{C}(z)-\mathbf{I}]=\mathscr{C}(z)-\mathbf{I}
$$


При этом же условии на порядок марковской цепи элементами матрицы $\mathbf{P D}^{-1} \mathbf{P}^{T}$ являются

$$
\begin{aligned}
& \sum_{k_{1}, \ldots, k_{m}} \delta_{\left(i_{2} \ldots i_{m}\right),\left(k_{1} \ldots k_{m-1}\right)} \delta_{\left(j_{2} \ldots j_{m}\right),\left(k_{1} \ldots k_{m-1}\right)} \frac{P\left(k_{m} \mid i_{1} \ldots i_{m}\right) P\left(k_{m} \mid j_{1} \ldots j_{m}\right)}{P\left(k_{1} \ldots k_{m}\right)} \\
& =\delta_{\left(i_{2} \ldots i_{m}\right),\left(j_{2} \ldots j_{m}\right)} \sum_{k_{m}} \frac{P^{2}\left(k_{m} \mid i_{2} \ldots i_{m}\right)}{P\left(i_{2} \ldots i_{m} k_{m}\right)} \\
& =\delta_{\left(i_{2} \ldots i_{m}\right),\left(j_{2} \ldots j_{m}\right)} \sum_{k_{m}} \frac{P\left(i_{2} \ldots i_{m} k_{m}\right)}{P^{2}\left(i_{2} \ldots i_{m}\right)}=\frac{\delta_{\left(i_{2} \ldots i_{m}\right),\left(j_{2} \ldots j_{m}\right)}}{P\left(i_{2} \ldots i_{m}\right)} .
\end{aligned}
$$

Тогда

$$
\mathbf{P D}^{-1} \mathbf{P}^{T}=\mathbf{V}
$$

Теорема 2.2. Если $\varepsilon_{1}, \ldots, \varepsilon_{n}$ образуют иепь Маркова порядка $m$ - 1, то имеют место Формуль (14) и (15), аде $\mathbf{V}$ задано в (12), а $\mathbf{D}=\operatorname{diag}(\mathbf{p})-$ диагональная матрица.

3. Производящие функции для цепочек с заданными частотами. Здесь $\Omega$ будет обозначать подмножество цепочек длины $m$. Наша цель - получить производящую функцию вероятностей $P_{G H}(n)=$ $P\left(E_{G H}(n)\right), G, H \in \Omega$, в марковской цепи $\varepsilon=\left(\varepsilon_{1}, \ldots, \varepsilon_{n}\right)$ длины $n$ и порядка $m$ для событий

$$
\begin{aligned}
E_{G H}(n)=\{ & G \varepsilon \text { кончается цепочкой } H ; \\
& G \text { и } H-\text { единственные элементы из } \Omega \text { в } G \varepsilon\} .
\end{aligned}
$$

Допускается, что $G$ или $H$ может совпадать с $\varnothing$. Если $G=\varnothing$, то $P_{\varnothing H}(n)=P\{H-$ единственный элемент из $\Omega$ в $\varepsilon$ и он стоит в конце $\varepsilon\}$. Тогда $P_{\varnothing H}(n)=0$, если $n<m$, и $P_{\varnothing H}(m)=P(H)$. Если $H=\varnothing$, то

$$
P_{G \varnothing}(n)=P\{\text { единственный элемент из } \Omega \text { в } G \varepsilon \text { есть } G\} \text {. }
$$

Отметим, что $P_{G \varnothing}(0)=1$, но $P_{G H}(0)=0$ для всех $G, H \in \Omega$. При $n<m$ неравенство $P_{G H}(n)>0$ означает, что $\left(G_{n+1} \ldots G_{m}\right)=\left(H_{1} \ldots H_{m-n}\right)$.

Положим

$$
F_{G H}(z)=\sum_{n=1}^{\infty} P_{G H}(n) z^{n},
$$

и пусть $\mathscr{F}(z)$ обозначает матрицу, образованную такими функциями. По определению вектор $\mathscr{F}_{1}(z)$ состоит из координат $F_{\varnothing H}(z)$, а вектор $\mathscr{F}_{2}(z)$ - из координат $F_{G \varnothing}(z)$. Матрица $\mathscr{C}_{\Omega}(z)$ получается из матрицы $\mathscr{C}(z)$ вычеркиванием строк и столбцов, не принадлежащих подмножеству $\Omega=\{A, B, \ldots, T\}$. Вектор вероятностей этих $m$-грамм обозначается через $\mathbf{p}_{\Omega}=(P(A), \ldots, P(T))^{T}$, и предполагается, что все такие вероятности положительны, а $\mathbf{I}_{\Omega}\left(\mathbf{e}_{\Omega}\right)$ обозначает здесь единичную матрицу (вектор единиц) соответствующей размерности. 
Теорема 3.1. Матрица $\mathscr{F}(z)$, образованная производящими функииями $F_{G H}, G, H \in \Omega$, такова, что

$$
\left[\mathbf{I}_{\Omega}-\mathscr{F}(z)\right]\left[\mathscr{C}_{\Omega}(z)+\frac{z^{m}}{1-z} \mathbf{e}_{\Omega} \mathbf{p}_{\Omega}^{T}\right]=\mathbf{I}_{\Omega}
$$

Кроме того,

$$
\begin{aligned}
{\left[\mathbf{I}_{\Omega}-\mathscr{F}(z)\right] \mathbf{e}_{\Omega} } & =(1-z) \mathscr{F}_{2}(z), \\
z^{m}\left[\mathbf{I}_{\Omega}-\mathscr{F}^{T}(z)\right] \mathbf{p}_{\Omega} & =(1-z) \mathscr{F}_{1}(z) .
\end{aligned}
$$

Д о к а з а т е л ь с т в о. Согласно (6), соотношение (17) означает, чTO

$$
\sum_{k=0}^{\infty} z^{k}\left[\mathbf{P}^{k}\right]_{\Omega}-\mathscr{F}(z) \sum_{k=0}^{\infty} z^{k}\left[\mathbf{P}^{k}\right]_{\Omega}=\mathbf{I}_{\Omega}
$$

Так как $\mathscr{F}(0)=0$, сравнение коэффициентов показывает, что ввиду (4) и (5) достаточно показать, что для всех $\ell=1, \ldots, m$ и $G, H \in \Omega$

$$
\begin{aligned}
& \delta_{\left(G_{\ell+1} \ldots G_{m}\right),\left(H_{1} \ldots H_{m-\ell}\right)} P\left(H_{m-\ell+1} \ldots H_{m} \mid G\right) \\
& \quad=\sum_{K \in \Omega} \sum_{n=1}^{\ell} P_{G K}(n) \delta_{\left(K_{\ell-n+1} \ldots K_{m}\right),\left(H_{1} \ldots H_{m-\ell+n}\right)} P\left(H_{m-\ell+n+1} \ldots H_{m} \mid K\right)
\end{aligned}
$$

и для $\ell=m+1, \ldots$

$$
\begin{aligned}
& P^{(\ell-m)}(H \mid G) \\
& =\sum_{K \in \Omega} \sum_{n=\ell-m+1}^{\ell} P_{G K}(n) \delta_{\left(K_{m-n+1} \ldots K_{m}\right),\left(H_{1} \ldots H_{n}\right)} P\left(H_{n+1} \ldots H_{m} \mid K\right) \\
& \quad+\sum_{K \in \Omega} \sum_{n=1}^{\ell-m-1} P_{G K}(n) P^{(\ell-m-n)}(H \mid K) .
\end{aligned}
$$

Чтобы доказать (20), заметим, что сумма в правой части этого равенства равна нулю, если $\left(G_{\ell+1} \ldots G_{m}\right) \neq\left(H_{1} \ldots H_{m-\ell}\right)$. Допустив последнее для любой цепочки $K$ из $\Omega$, имеем

$$
\sum_{n=1}^{\ell} P_{G K}(n) \delta_{\left(K_{\ell-n+1} \ldots K_{m}\right),\left(H_{1} \ldots H_{m-\ell+n}\right)} P\left(H_{m-\ell+n+1} \ldots H_{m} \mid K\right)=0
$$

если только при некотором $n \leqslant l\left(K_{\ell-n+1} \ldots K_{m}\right)=\left(H_{1} \ldots H_{m-\ell+n}\right)$. Тогда, согласно нашему предположению, $\left(K_{\ell-n+1} \ldots K_{m-n}\right) \neq\left(G_{\ell+1} \ldots G_{m}\right)$, так что $\left(K_{1} \ldots K_{m-n}\right) \neq\left(G_{n+1} \ldots G_{m}\right)$, и $P_{G K}(n)=0$. Таким образом, при доказательстве $(20)$ можно считать, что $\left(G_{\ell+1} \ldots G_{m}\right)=$ $\left(H_{1} \ldots H_{m-\ell}\right)$. 
Рассмотрим последовательность $\left(G_{1} \ldots G_{m} H_{m-l+1} \ldots H_{m}\right)=\left(G_{1} \ldots\right.$ $\left.G_{\ell} H_{1} \ldots H_{m}\right)$ длины $m+l$, вероятность іоявления которой, очевидно, равна $P\left(H_{m-l+1} \ldots H_{m} \mid G\right) P(G)$. Пусть $K$ является первым после $G$ элементом $\Omega$ в этой последовательности, обозначим занимаемый им номер через $n$. Тогда $\left(K_{m-n+1} \ldots K_{m}\right)=\left(H_{m-\ell+1} \ldots H_{m-l+n}\right)$ и

$$
\begin{aligned}
& P\left(G_{1} \ldots G_{m} H_{m-l+1} \ldots H_{m}\right) \\
& =\sum_{K \in \Omega} \sum_{n=1}^{l} P(G) P_{G K}(n) P\left(H_{m-\ell+n+1} \ldots H_{m} \mid K\right) \\
& =\sum_{K \in \Omega} \sum_{n=1}^{l} \delta_{\left(K_{m-n+1} \ldots K_{m}\right),\left(H_{m-l+1} \ldots H_{m-\ell+n}\right)} P(G) \\
& \quad \times P_{G K}(n) P\left(H_{m-\ell+n+1} \ldots H_{m} \mid K\right) .
\end{aligned}
$$

Тем самым (20) доказано; аналогично доказывается (21).

Рассмотрим множество всех $\ell$-грамм, начинающихся с $G$ и кончающихся $H$. Пусть место первого появления в такой $\ell$-грамме цепочки из $\Omega$, скажем, $K$, имеет номер $n$. Если $n<\ell-m$, то вторая сумма в (21) представляет соответствуюшую вероятность, а при $n \geqslant \ell-m$ эту вероятность дает первая сумма.

Формула (18) близка уравнениям теоремы 3.3 в [6, с. 192]. Так как $P_{G \varnothing}(0)=1$ и $P_{G H}(0)=0$, она означает, что

$$
P_{G \varnothing}(n)=P_{G \varnothing}(n+1)+P_{G A}(n+1)+\cdots+P_{G T}(n+1),
$$

и следует из того, что событие $E_{G \varnothing}(n)$, определяющее $P_{G \varnothing}(n)$, является объединением по добавлению последней буквы к $n$-грамме. Каждая из таких $(n+1)$-грамм может кончаться любым элементом $\Omega$ или же ни одним из них.

Для доказательства последнего уравнения (19) заметим, что, согласно этому уравнению, для $n=m, m+1, \ldots$

$P_{\varnothing G}(n)=P_{\varnothing G}(n+1)+P(A) P_{A G}(n+1-m)+\cdots+P(T) P_{T G}(n+1-m)$.

В самом деле, событие $E_{\varnothing G}(n)$ является объединением по первой букве, к которой присоединяются $n$-граммы. Каждая из таких $(n+1)$-грамм может начинаться с одного из элементов $\Omega$ или же ни с какого. Теорема 3.1 доказана.

Теорема 3.1 при $\Omega=\mathscr{I}_{m}$ показывает, что $\mathscr{F}(z)=z \mathbf{P}$. Действительно, в этом случае, согласно (6),

$$
\mathbf{I}_{q^{m}}-\mathscr{F}(z)=\left[\mathscr{C}(z)+\frac{z^{m}}{1-z} \mathbf{e p}^{T}\right]^{-1}=\mathbf{I}_{q^{m}}-z \mathbf{P}
$$

что несложно проверить непосредственно. 
Частный случай теоремы 3.1, когда порядок цепи равен единице, содержится в [15], где получена формула для производящей функции вероятностей того, что $m$-грамма $\imath$ появится определенное число раз, скажем, $r$, где $r \geqslant 1$. Эта формула является следствием теоремы 3.1 при $\Omega=\{\imath\}$.

Как было отмечено в п. 2, функция

$$
(1-z) \mathscr{C}_{\imath \jmath}(z)+z^{m} P(\jmath)=\frac{H_{\imath \jmath}(z)}{G(z)}
$$

является отношением двух многочленов

$$
H_{\imath \jmath}(z)=(1-z) A_{\jmath \imath}(z)+z P(\jmath) G(z)
$$

где $A_{\jmath \imath}$ есть алгебраическое дополнение в матрице $\left[\mathbf{I}_{q^{m}}-z\left(\mathbf{P}-\mathbf{e p}^{T}\right)\right]^{-1}$, и $G(z)=\operatorname{det}\left(\mathbf{I}_{q^{m}}-z\left(\mathbf{P}-\mathbf{e p}^{T}\right)\right)=\operatorname{det}\left(\mathbf{I}_{q^{m}}-z \mathbf{P}\right) /(1-z)$. Очевидно, что $H_{\imath \jmath}(z)$ есть алгебраическое дополнение в матрице $\left(\mathbf{I}_{q^{m}}-z \mathbf{P}\right)^{-1}$. Если $F_{\imath \imath}(z)$ обозначает производящую функцию из теоремы 3.1 , то

$$
F_{\imath}^{r}(z)=\sum_{n=0}^{\infty} z^{n} P\left\{\imath \text { появляется в } \varepsilon_{n} \text { ровно } r \text { раз }\right\}=\mathscr{F}_{1}(z)\left[F_{\imath \imath}(z)\right]^{r-1} \mathscr{F}_{2}(z) .
$$

Согласно теореме 3.1 ,

$$
F_{\imath \imath}(z)=\frac{(1-z)\left(\mathscr{C}_{\imath \imath}(z)-1\right)+z^{m} P(\imath)}{(1-z) \mathscr{C}_{\imath \imath}(z)+z^{m} P(\imath)}
$$

так что

$$
\begin{aligned}
F_{\imath}^{r}(z) & =\frac{z^{m} P(\imath)\left[(1-z)\left(\mathscr{C}_{\imath \imath}(z)-1\right)+z^{m} P(\imath)\right]^{r-1}}{\left[(1-z) \mathscr{C}_{\imath \imath}(z)+z^{m} P(\imath)\right]^{r+1}} \\
& =\frac{z^{m} P(\imath)[G(z)]^{2}\left[H_{\imath \imath}(z)-(1-z) G(z)\right]^{r-1}}{\left[H_{\imath \imath}(z)\right]^{r+1}} .
\end{aligned}
$$

Если $r=0$, то

$$
F_{\imath}^{0}(z)=\frac{1-\mathscr{F}_{1}(z)}{1-z}=\frac{A_{\imath \imath}(z)}{(1-z) \mathscr{C}_{\imath \imath}(z)+z^{m} P(\imath)}=\frac{H_{\imath \imath}(z)-z^{m} P(\imath) G(z)}{(1-z) H_{i i}(z)} .
$$

Впервые формулы такого рода были получены А. Д. Соловьевым [18].

Нам понадобится частный случай теоремы 3.1 для множества $\Omega$, состоящего из двух элементов.

Теорема 3.2. Пусть $\Omega=\{\imath, \jmath\}, a$

$$
\begin{aligned}
B_{\imath \jmath}(z)= & (1-z)|\mathscr{C}(z)| \\
& +z^{m}\left[P(\jmath) \mathscr{C}_{\imath \imath}(z)+P(\imath) \mathscr{C}_{\jmath \jmath}(z)-P(\imath) \mathscr{C}_{\imath \jmath}(z)-P(\jmath) \mathscr{C}_{\jmath \imath}(z)\right]
\end{aligned}
$$


где $\mathscr{C}_{\imath \jmath}(z)$ задано в (2) и $|\mathscr{C}(z)|=\mathscr{C}_{\imath \imath}(z) \mathscr{C}_{\jmath \jmath}(z)-\mathscr{C}_{\imath \jmath}(z) \mathscr{C}_{\jmath \imath}(z)$. При $H_{\imath \jmath}$, определенном в (22),

$$
B_{\imath \jmath}(z)=(1-z)^{3} G^{2}(z)\left[\frac{1}{H_{\imath \imath}(z) H_{\jmath \jmath}(z)}-\frac{1}{H_{\imath \jmath}(z) H_{\jmath \imath}(z)}\right]
$$

является рациональной функцией, а

$$
\begin{aligned}
F_{\imath \varnothing}(z) & =\frac{\mathscr{C}_{\jmath \jmath}(z)-\mathscr{C}_{\imath \jmath}(z)}{B_{\imath \jmath}(z)}, \\
F_{\varnothing_{\imath}}(z) & =\frac{z^{m}\left[P(\imath) \mathscr{C}_{\jmath \jmath}(z)-P(\jmath) \mathscr{C}_{\jmath \imath}(z)\right]}{B_{\imath \jmath}(z)}, \\
F_{\imath \jmath}(z) & =\frac{(1-z) \mathscr{C}_{\imath \jmath}(z)+z^{m} P(\jmath)}{B_{\imath \jmath}(z)} \\
F_{\imath \imath}(z) & =1-\frac{(1-z) \mathscr{C}_{\jmath \jmath}(z)+z^{m} P(\jmath)}{B_{\imath \jmath}(z)}
\end{aligned}
$$

и соответствуюшие формуль верньи для $F_{\jmath \varnothing}, F_{\varnothing \jmath}, F_{\jmath \imath}$ и $F_{\jmath \jmath}$.

Так же как в [12], теорема 3.2 может быть использована для получения явного вида рациональной производящей функции $F_{\imath \jmath}^{r t}(z)$ вероятностей того, что частоты двух цепочек $\imath$ и $\jmath$ в последовательности длины $n$ равны $r$ и $t$.

4. Асимптотическая формула для математического ожидания числа цепочек, появляющихся заданное число раз. Формулы для производящих функций дают асимптотику математического ожидания числа $m$-цепочек, появляюшихся заданное число раз, а также их ковариаций. Предположим, что $n \rightarrow \infty$ и $q \rightarrow \infty$ таким образом, что $n / q^{m} \rightarrow \alpha$ для положительного $\alpha$. Для обоснования такой асимптотики в случае бинарных последовательностей длины $n$ надо выбрать натуральное $p$ и объявить все $p$-цепочки, образованные нулями и единицами, буквами нового алфавита кардинальности $q=2^{p}$. Тогда новым $m$-граммам соответствуют изначальные непересекающиеся $2 m$-цепочки, $\alpha=n / q^{m}=n / 2^{m p}$. Изучая тестирование случайности, обычно предполагают, что распределение букв близко к равномерному в том смысле, что для любой $m$-граммы $\imath$ верно $P(j \mid \imath)-q^{-1} \sim q^{-s}$ с некоторым $s>1$ и выбор $s$ может зависеть от $m$. Так, в критерии пустых ящиков разумно положить $s=m / 4+1$ (см. [21]). П.Ф. Беляев [2] предложил схожие условия еще в 1965 г., они также используются в монографии [8].

Мы предположим, что для всякой $m$-цепочки $\imath$ выполняется $P(j \mid \imath)=q^{-1}+\psi_{\imath j} q^{-3 / 2}$, где $\psi_{\imath j}$ - равномерно ограниченная последовательность такая, что $\sum_{j=1}^{q} \psi_{\imath j}=0$. Для $k=0,1, \ldots, m-1$ положим

$$
\psi_{\ldots j_{k+1} \ldots i_{m-1} j_{m}}=\frac{1}{q^{k+1}} \sum_{j_{0}, j_{1}, \ldots, j_{k}} \psi_{\left(j_{0} \ldots j_{m-1}\right) j_{m}} .
$$


Предполагается, что при $k=0,1, \ldots, m-1$

$$
\frac{1}{q^{m-k}} \sum_{j_{k+1}, \ldots, j_{m}} \psi_{\cdots j_{k+1} \ldots j_{m-1} j_{m}}^{2} \longrightarrow \mathbf{D}_{k}
$$

и $\mathbf{D}=\sum_{k=0}^{m-1} \mathbf{D}_{k}>0$. В матричной форме $\mathbf{P}=\mathbf{P}_{0}+\Psi$, где $\mathbf{P}_{0}$ состоит из элементов вида $\delta_{\left(i_{2} \ldots i_{m}\right),\left(j_{1} \ldots j_{m-1}\right)} q^{-1}$, а матрица $\Psi$ - из элементов $\delta_{\left(i_{2} \ldots i_{m}\right),\left(j_{1} \ldots j_{m-1}\right)} q^{-3 / 2} \psi_{\imath j_{m}}, \Psi \mathbf{e}=0$. Тогда вектор стационарных вероятностей $\mathbf{p}$ имеет вид

$$
\mathbf{p}=\frac{1}{q^{m}}\left[\mathbf{I}-\sum_{\ell=0}^{m-1}\left[\mathbf{P}_{0}^{T}\right]^{\ell} \Psi^{T}\right]^{-1} \mathbf{e} .
$$

В самом деле,

$$
\mathbf{p}=\left[\mathbf{I}-\left(\mathbf{I}-\mathbf{P}_{0}^{T}+q^{-m} \mathbf{e e}^{T}\right)^{-1} \Psi^{T}\right]^{-1} \frac{\mathbf{e}}{q^{m}},
$$

так как

$$
\begin{aligned}
\mathbf{I} & -\Psi\left(\mathbf{I}-\mathbf{P}_{0}+q^{-m} \mathbf{e e}^{T}\right)^{-1}=\left(\mathbf{I}-\mathbf{P}_{0}+q^{-m} \mathbf{e e}^{T}-\Psi\right)\left(\mathbf{I}-\mathbf{P}_{0}+q^{-m} \mathbf{e e}^{T}\right)^{-1} \\
& =\left(\mathbf{I}-\mathbf{P}+q^{-m} \mathbf{e e}^{T}\right)\left(\mathbf{I}-\mathbf{P}_{0}+q^{-m} \mathbf{e e}^{T}\right)^{-1} \\
& =\left(\mathbf{I}-\mathbf{P}+\mathbf{e} p^{T}\right)\left(\mathbf{I}+q^{-m} \mathbf{e} \mathbf{e}^{T}-\mathbf{e} p^{T}\right)\left(\mathbf{I}-\mathbf{P}_{0}+q^{-m} \mathbf{e e}^{T}\right)^{-1},
\end{aligned}
$$

a

$$
\left(\mathbf{I}-\mathbf{P}_{0}^{T}+q^{-m} \mathbf{e e}^{T}\right)^{-1}\left(\mathbf{I}+q^{-m} \mathbf{e e}^{T}-\mathbf{p e}^{T}\right)\left(\mathbf{I}-\mathbf{P}^{T}+\mathbf{p e}^{T}\right) \mathbf{p}=\frac{\mathbf{e}}{\mathbf{q}^{\mathbf{m}}} .
$$

Поскольку $\mathbf{P}_{0}^{m}=q^{-m} \mathbf{e e}^{T}$, получаем (24), так что при

$\xi_{\imath}=\sum_{k=0}^{m-1} \psi_{\ldots i_{1} \ldots i_{m-k-1} i_{m-k}} \quad$ и $\quad \Delta_{\imath}=\frac{1}{q} \sum_{k=0}^{m-1} \sum_{j} \psi_{\left(j i_{1} \ldots i_{m-1}\right) i_{m}} \psi_{\ldots j i_{1} \ldots i_{m-k-2} i_{m-k-1}}$

имеем

$$
P(\imath)=\frac{1}{q^{m}}\left[1+\frac{\xi_{\imath}}{q^{1 / 2}}+\frac{\Delta_{\imath}}{q}+O\left(\frac{1}{q^{3 / 2}}\right)\right] .
$$

Таким образом, при наших условиях

a

$$
e^{-n P(\imath)}=e^{-\alpha}\left[1-\frac{\alpha \xi_{\imath}}{q^{1 / 2}}+\frac{\alpha^{2} \xi_{2}^{2}-2 \alpha \Delta_{\imath}}{2 q}+O\left(\frac{1}{q^{3 / 2}}\right)\right],
$$

$$
[n P(\imath)]^{r}=\alpha^{r}\left[1+\frac{r \xi_{2}}{q^{1 / 2}}+\frac{r(r-1) \xi_{2}^{2}+2 r \Delta_{2}}{2 q}+O\left(\frac{1}{q^{3 / 2}}\right)\right] .
$$

Аналогично (24) выводим

$$
\begin{aligned}
(\mathbf{I}- & \left.\mathbf{P}+\mathbf{e p}^{T}\right)^{-1}=\left(\mathbf{I}-\mathbf{P}_{0}+q^{-m} \mathbf{e e}^{T}\right)^{-1}\left[\mathbf{I}-\Psi\left(\mathbf{I}-\mathbf{P}_{0}+q^{-m} \mathbf{e e}^{T}\right)^{-1}\right]^{-1} \\
& -q^{-m} \mathbf{e e}^{T}\left[\mathbf{I}-\Psi\left(\mathbf{I}-\mathbf{P}_{0}+q^{-m} \mathbf{e e}^{T}\right)^{-1}\right]^{-1} \Psi\left(\mathbf{I}-\mathbf{P}_{0}+q^{-m} \mathbf{e e}^{T}\right)^{-1} \\
& \times\left(\mathbf{I}-\mathbf{P}_{0}+q^{-m} \mathbf{e e}^{T}\right)^{-1}\left[\mathbf{I}-\Psi\left(\mathbf{I}-\mathbf{P}_{0}+q^{-m} \mathbf{e e}^{T}\right)^{-1}\right]^{-1} .
\end{aligned}
$$


Пусть $s=s(\imath), s \geqslant 1$, обозначает период цепочки $\imath$, т.е. наименьшее натуральное число, для которого $\left(i_{s+1} \ldots i_{m}\right)=\left(i_{1} \ldots i_{m-s}\right)$. Для периодических цепочек

$$
\begin{aligned}
\left(\mathbf{I}-\mathbf{P}+\mathbf{e p}^{T}\right)_{\imath \imath}^{-1}= & \left.1+\frac{1}{q^{s}}+\frac{1}{q^{s+1 / 2}} \sum_{r=0}^{s-1} \psi_{\left(i_{r+1} \ldots i_{s} i_{1} \ldots i_{m-s+r}\right.}\right) i_{m-s+r+1} \\
& +O\left(\frac{1}{q^{s+3 / 2}}\right) .
\end{aligned}
$$

В отсутствие периода полагаем $s=\infty$, и тогда

$$
\left(\mathbf{I}-\mathbf{P}+\mathbf{e p}^{T}\right)_{\imath 2}^{-1}=1+O\left(\frac{1}{q^{m}}\right) .
$$

Для асимптотики вероятности $\pi_{\imath}^{r}(n)$ того, что цепочка $\imath$ появляется в последовательности длины $n$ заданное число раз, можно использовать формулы (A1)-(A4) из [17]. Производящая функция $F_{2}^{r}(z)$ для этих вероятностей указана в (23). При наших условиях единственный наименьший корень $\widehat{z}=z_{1}$ уравнения $H_{\imath \imath}(z)=0$, который и определяет поведение рациональной функции (23), вешественен, $\widehat{z}>1, \widehat{z} \rightarrow 1$, когда $q \rightarrow \infty$. Все другие (комплексные) корни $z_{k}$ стремятся к бесконечности, $\min _{k \geqslant 2}\left|z_{k}\right| \geqslant q$, что может быть усмотрено из поведения резольвенты, связывающей $\left[\mathbf{I}-z\left(\mathbf{P}-\mathbf{e p}^{T}\right)\right]^{-1} \mathrm{c}\left[\mathbf{I}-z\left(\mathbf{P}_{0}-q^{-m} \mathbf{e e}^{T}\right)\right]^{-1}$.

При $r=0$ можно использовать результат из [12], согласно которому

$$
\pi_{\imath}^{0}(n)=-\frac{A_{\imath \imath}(\widehat{z})}{H_{\imath \imath}^{\prime}(\widehat{z}) \widehat{z}^{n+1}}+O\left(\frac{1}{q^{n}}\right)
$$

Для $r \geqslant 1$ и $n \geqslant m$

$$
\pi_{\imath}^{r}(n)=(-1)^{r+1} \frac{P(\imath)[G(\widehat{z})]^{r+1}(\widehat{z}-1)^{r-1}}{\left[H_{\imath \imath}^{\prime}(\widehat{z})\right]^{r+1} \widehat{z}^{n-m+r}}\left(\begin{array}{c}
r+n-m \\
r
\end{array}\right)+O\left(\frac{1}{q^{n}}\right) .
$$

Согласно определению $H_{\imath \imath}$,

$$
(\widehat{z}-1)\left[\mathbf{I}-\widehat{z}\left(\mathbf{P}-\mathbf{e p}^{T}\right)\right]_{\imath \imath}^{-1}=\widehat{z} P(\imath)
$$

Tak кaK

$$
\left[\mathbf{I}-\widehat{z}\left(\mathbf{P}-\mathbf{e p}^{T}\right)\right]_{\imath \imath}^{-1}=\left(\mathbf{I}-\mathbf{P}+\mathbf{e p}^{T}\right)_{\imath \imath}^{-1}+O\left(\frac{1}{q^{m}}\right)
$$

TO

$$
\widehat{z}=1+P(\imath)-q^{-s} P(\imath)+O\left(\frac{1}{q^{m+s+1 / 2}}\right)
$$

Кроме того,

$$
H_{\imath \imath}^{\prime}(\widehat{z})=-\frac{H_{\imath \imath}(1)}{\widehat{z}-1}+O\left(\frac{1}{q^{m}}\right)
$$


Итак, для всех $r$

$$
\begin{aligned}
\pi_{\imath}^{r}(n)= & \frac{P(\imath)[G(1)]^{r+1}(\widehat{z}-1)^{2 r} n^{r}}{\left[H_{\imath \imath}(1)\right]^{r+1} \widehat{z}^{n+r+1}}+O\left(\frac{1}{q^{n}}\right)=\frac{\alpha^{r} e^{-\alpha}}{r !} \\
& \times\left[1+\frac{(r-\alpha) \xi_{\imath}}{q^{1 / 2}}+\frac{\left(\alpha^{2}-2 \alpha r+r(r-1)\right) \xi_{\imath}^{2}+2(r-\alpha) \Delta_{\imath}}{2 q}\right. \\
& \left.+\frac{\alpha^{2}-2 \alpha r+r(r-1)}{\alpha q^{s}}+O\left(\frac{1}{q^{3 / 2}}\right)\right] .
\end{aligned}
$$

Заметим, что $\sum_{\imath} \xi_{\imath}=\sum_{\imath} \Delta_{\imath}=0$, а $\sum_{\imath} \xi_{\imath}^{2}=\mathbf{e}^{T} \Psi \sum_{k=0}^{m-1} \mathbf{P}_{0}^{k} \sum_{\ell=0}^{m-1}\left[\mathbf{P}_{0}^{T}\right]^{\ell} \Psi^{T} \mathbf{e}$. Координаты вектора $\Psi^{T}$ е суть $\psi_{\cdot j_{1} \ldots j_{m-1} j_{m}}$, матрица $\left(\mathbf{P}_{0} \mathbf{P}_{0}^{T}\right)^{k}$ образована элементами $\delta_{\left(i_{k+1} \ldots i_{m}\right),\left(j_{k+1} \ldots j_{m}\right)} q^{-k}$, а для $k \neq \ell$ верно $\mathbf{e}^{T} \Psi \mathbf{P}_{0}^{k}\left[\mathbf{P}_{0}^{T}\right]^{\ell} \Psi^{T} \mathbf{e}=0$. Тем самым

$\sum_{\imath} \xi_{\imath}^{2}=\mathbf{e}^{T} \Psi\left[\sum_{k=0}^{m-1}\left(\mathbf{P}_{0} \mathbf{P}_{0}^{T}\right)^{k}\right] \Psi^{T} \mathbf{e}=\sum_{k=0}^{m-1} q^{k}\left[\sum_{j_{k+1}, \ldots, j_{m}} \psi_{\cdots j_{k+1} \ldots j_{m-1} j_{m}}^{2}\right] \sim q^{m} \mathbf{D}$.

Формула (25) приводит к выражению для математического ожидания числа $N^{r}=N_{n}^{r} m$-грамм, появляющихся ровно $r$ раз в $n$ последовательности. При $r=0,1,2, \ldots$

$E N^{r}=\sum_{\imath} \pi_{\imath}^{r}(n)=\frac{\alpha^{r} e^{-\alpha}}{r !} q^{m}\left[1+\frac{\mathrm{D}}{2 q}\left[\alpha^{2}-2 \alpha r+r(r-1)\right]+O\left(\frac{1}{q^{3 / 2}}\right)\right]$.

Для независимых одинаково распределенных последовательностей имеем $\psi_{\imath j}=\psi_{j}$, так что $\mathbf{D}_{k} \equiv \mathbf{D}_{m-1}=q^{-1} \sum_{j=1}^{q} \psi_{j}^{2}$, а $\mathbf{D}=m \mathbf{D}_{m-1}$. В случае $r=0$ (26) согласуется с формулой на с. 23 работы М.И. Тихомировой и В. П. Чистякова [21], полученной этими авторами другим методом.

Асимптотическое разложение ковариации между $N^{r}$ и $N^{t}$ может быть получено согласно формулам (А9), (А10) в [17]. При $r \neq t$

$$
\begin{aligned}
\operatorname{Cov}\left(N^{r}, N^{t}\right)= & -q^{m} \frac{e^{-2 \alpha} \alpha^{r+t}}{r ! t !}[(2 m-1) \alpha-(2 m-1)(r+t)+1 \\
& \left.+\frac{(4 m-3) r t}{\alpha}-\frac{2(m-1)(\alpha-r)(\alpha-t)}{\alpha}\right]+O\left(q^{m-1}\right) \\
= & -q^{m} \frac{e^{-2 \alpha} \alpha^{r+t}}{r ! t !}\left[\alpha-r-t+1+\frac{r t}{\alpha}\right]+O\left(q^{m-1}\right),
\end{aligned}
$$

a

$$
\operatorname{Var}\left(N^{r}\right)=q^{m} \frac{e^{-\alpha} \alpha^{r}}{r !}\left[1-\frac{e^{-\alpha} \alpha^{r}}{r !}\left(\alpha-2 r+1+\frac{r^{2}}{\alpha}\right)\right]+O\left(q^{m-1}\right) .
$$

Теорема 4.1. Если $n \rightarrow \infty u n / q^{m} \rightarrow \alpha$ с положительнылм $\alpha$, то

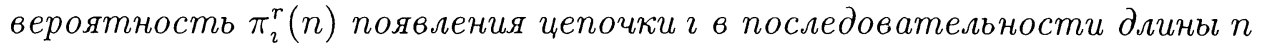


ровно $r$ раз допускает асимптотическое разложение (25). Для математического ожидания числа чепочек, появляющихся $r$ раз, справедлива формула (26). Ковариаиия между числами чепочек, появляющихся $r$ и $t$ раз имеет вид (27), а дисперсия чепочек с частотой $r$ представима как (28).

В теореме 6 главы III книги [8] приводятся формулы для двух первых моментов распределения числа цепочек с заданной частотой в предположении, что появления цепочек независимы, т.е. подсчитываются непересекающиеся $m$-цепочки. Заслуживает некоторого удивления тот факт, что асимптотика моментов одна и та же для налагающихся и неналагающихся цепочек.

По этой причине оптимальный линейный критерий, основанный на статистике отсутствующих цепочек, цепочек частоты один, два и т.д. в марковской последовательности, тот же, что и тест, данный в теореме 2 главы V книги [8]. В. Г. Михайлов [10], [11] изучал условия предельной нормальности для таких статистик.

5. Пример: 2-граммы. Приближения для математического ожидания и дисперсии числа отсутствующих пар изучены в [9] и более тщательно в [20]. На этих приближениях основывается распределение одного теста случайности в [16]. Начнем обсуждение с цепи Маркова порядка два.

Согласно теореме 2.1 , вероятность $\pi_{\imath}^{0}(n)$ того, что $n$-последовательность не содержит $\imath=\left(i_{1}, i_{2}\right)$, есть коэффициент при $z^{n}$ разложения в степенной ряд функции

$$
\frac{H_{\imath \imath}(z)-z^{2} P(\imath) G(z)}{(1-z) H_{\imath \imath}(z)}=\frac{1}{1-z}-\frac{z^{2} P(\imath)}{(1-z)^{2}(\mathbf{I}-z \mathbf{P})_{\imath \imath}},
$$

где $H_{\imath \imath}(z)$ обозначает алгебраическое дополнение элемента $(\imath, \imath)$ матрицы $\mathbf{I}-z \mathbf{P}(\mathbf{P}$ определено в $(3))$, a $G(z)=\operatorname{det}(\mathbf{I}-z \mathbf{P}) /(1-z)$. Пусть алфавит состоит из $q=2$ букв, скажем, 0 и 1 , и положим $a=P(1 \mid 00), b=$ $P(1 \mid 01), c=P(0 \mid 10), d=P(0 \mid 11)$. Тогда

$$
\begin{aligned}
H_{(0,0)(0,0)}(z)= & 1-z(1-d)-z^{2}(1-b)(1-c)+z^{3}(1-b-d)(1-c) \\
H_{(0,1)(0,1)}(z)= & (1-(1-a) z)(1-(1-d) z) \quad \text { и т.п. и } \\
G(z)= & (1-a-c)(1-b-d) z^{3}+(a d-(1-b)(1-c)) z^{2} \\
& -(1-a-d) z+1 .
\end{aligned}
$$

И. Дж. Гуд [5] подсчитал стационарные вероятности $P(00)=c d /(a b+$ $c d+2 a d), P(11)=a b /(a b+c d+2 a d), P(10)=P(01)$ и указал формулу для дисперсии числа отсутствующих цепочек.

Например, наша формула для $H_{(0,0)(0,0)}(z)$ показывает, что

$$
\pi_{(00)}^{0}(n)=\frac{c d(a b+d-a)(1-a)^{n-3}-a b(c d-d+a)(1-d)^{n-3}}{(d-a)(a b+c d+2 a d)} .
$$


Полученные производящие функции дают формулы для вероятностей частот заданной цепочки в марковских цепях порядка два (ср. [3]).

Если же цепь имеет порядок один с переходной матрицей П, то, согласно теореме 2.1 , при $m=2$ для любого $\imath=\left(i_{1}, i_{2}\right)$

$(1-z) C_{\imath \imath}(z)+z^{2} P(\imath)=(1-z)\left[1+\delta_{i_{1} i_{2}} z \Pi_{i_{1} i_{2}}+z^{2} \Pi_{i_{1} i_{2}}\left[\Pi(\mathbf{I}-z \Pi)^{-1}\right]_{i_{2} i_{1}}\right]$, а для $\imath \neq \jmath$

$$
C_{\imath \jmath}(z)=\delta_{i_{2} j_{1}} z \Pi_{j_{1} j_{2}}+z^{2} \Pi_{j_{1} j_{2}}\left[\mathbf{I}-z\left(\Pi-\mathbf{e} \pi^{T}\right)\right]_{i_{2} j_{1}}^{-1}-z^{2} P(\jmath) .
$$

Формула для $\pi_{\imath}^{0}(n)$ значительно упрощается, когда $1-a=c, 1-b=d$. Если $n \rightarrow \infty$ и $q \rightarrow \infty$ так, что $n / q^{m} \rightarrow \alpha>0$, и $\Pi(j \mid i)=q^{-1}+q^{-3 / 2} \psi_{i j}$,

$$
\frac{1}{q^{2}} \sum_{i, j} \psi_{i j}^{2}=\mathbf{D}_{0}, \quad \frac{1}{q} \sum_{j} \psi_{\cdot j}^{2}=\mathbf{D}_{1}
$$

то $\pi(j)=q^{-1}+q^{-5 / 2} \sum_{i} \psi_{i j}=q^{-1}+q^{-3 / 2} \psi \cdot j$. Как и ранее, получаем

$$
n P(\imath)=\alpha\left[1+\frac{1}{q^{1 / 2}}\left(\psi_{i_{1} i_{2}}+\psi_{\cdot i_{1}}\right)+\frac{1}{q} \psi_{i_{1} i_{2}} \psi_{\cdot i_{1}}+O\left(\frac{1}{q^{2}}\right)\right] .
$$

Для апериодических цепочек $\imath=\left(i_{1}, i_{2}\right), i_{1} \neq i_{2}$, справедливы соотношения

$$
\begin{aligned}
\pi_{\left(i_{1}, i_{2}\right)}= & e^{-\alpha}-\frac{\alpha e^{-\alpha}}{\sqrt{q}}\left(\psi_{i_{1} i_{2}}+\psi_{\cdot i_{1}}\right) \\
& +\frac{\alpha e^{-\alpha}}{2 q}\left[\alpha\left(\psi_{i_{1} i_{2}}+\psi_{\cdot i_{1}}\right)^{2}-2 \psi_{i_{1} i_{2}} \psi_{\cdot i_{1}}\right]+O\left(\frac{1}{q^{2}}\right)
\end{aligned}
$$

а для периодических $(i, i)$

$\pi_{(i, i)}=e^{-\alpha}-\frac{\alpha e^{-\alpha}}{\sqrt{q}}\left(\psi_{i i}+\psi_{\cdot i}\right)+\frac{\alpha e^{-\alpha}}{2 q}\left[\alpha\left(\psi_{i i}+\psi_{\cdot i}\right)^{2}-2 \psi_{i i} \psi_{\cdot i}+2\right]+O\left(\frac{1}{q^{3 / 2}}\right)$.

Имеем $\sum_{i_{1}, i_{2}}\left(\psi_{i_{1} i_{2}}+\psi_{\cdot i_{1}}\right)^{2}=q^{2}\left[\mathbf{D}_{0}+\mathbf{D}_{1}\right], \sum_{i_{1}, i_{2}} \psi_{i_{1} i_{2}} \psi_{i_{1}}=0$, так что среднее числа отсутствующих цепочек $N^{0}$ имеет вид

$$
E N^{0}=e^{-\alpha} q^{2}\left[1+\frac{\alpha\left(\mathbf{D}_{0}+\mathbf{D}_{1}\right)}{2 q}+\frac{\alpha}{q^{2}}+o\left(\frac{1}{q^{3}}\right)\right] .
$$

Дисперсия $N^{0}$ приобретает вид

$$
\begin{aligned}
\operatorname{Var}\left(N^{0}\right)= & e^{-\alpha}\left[1-(1+\alpha) e^{-\alpha}\right] q^{2}+\alpha^{2} e^{-2 \alpha} q \\
& +e^{-\alpha}\left[1-\alpha-e^{-\alpha}\left(2-8 \alpha-\frac{3 \alpha^{2}}{2}\right)\right]\left(\mathbf{D}_{0}+\mathbf{D}_{1}\right) q+O(1) .
\end{aligned}
$$

Когда $\mathbf{D}_{0}+\mathbf{D}_{1}=0$, имеем значительно более точную формулу

$$
\begin{aligned}
\operatorname{Var}\left(N^{0}\right)= & e^{-\alpha}\left[1-(1+\alpha) e^{-\alpha}\right] q^{2}+\alpha^{2} e^{-2 \alpha} q \\
& +e^{-\alpha}\left[1-\frac{\alpha}{2}-e^{-\alpha}\left(1+2 \alpha-\frac{\alpha^{2}}{2}-\frac{\alpha^{3}}{2}\right)\right]+O\left(\frac{1}{q}\right) .
\end{aligned}
$$


Приближения (30), (31) ведут к удивительно точным численным результатам. Так, $E N^{0}=141909.3299555$, в то время как (30) дает 141909.3299551.

Эффективность критерия числа пустых ящиков задается выражением $\alpha^{2}\left[e^{\alpha}-1-\alpha\right]^{-1 / 2}$. Его максимум (соответствующий наиболее мощному тесту) получается, когда $\alpha=\alpha^{\star}=3.594$. Таким образом, при $m=2$ оптимальное соотношение между $q$ и $n$ есть $n \approx 3.6 q^{2}$. В этой ситуации критерий, основанный на $N^{0}$, асимптотически эффективен.

\section{6. Ковариационная матрица частот цепочек и ее псевдо-} обратная. Здесь исследуется связь ковариационной матрицы эмпирических частот $m$-грамм в цепи Маркова порядка $m$ с корреляционной матрицей цепочек $\mathscr{C}$. Пусть $Y_{n}$ обозначает обычную цепь Маркова, состояниями которой являются все $m$-граммы [1]. Более точно, $Y_{n}=\imath$, когда $\left(\varepsilon_{n}, \ldots, \varepsilon_{n+m-1}\right)=\left(i_{1} \ldots i_{m}\right)$.

Переходные вероятности этой цепи задаются формулой (3). Частота $\omega_{\imath}$ появления граммы $\imath$ в изначальной последовательности равна частоте состояния $\imath$ новой цепи.

Хорошо известно [7], что совместное распределение таких частот асимптотически нормально. Переходная матрица $\mathbf{P}$, заданная в (3), имеет единственное собственное значение, по модулю равное 1 (так что цепь регулярна). Такая матрица $\mathbf{P}$ имеет вектор стационарных вероятностей $\mathbf{p}$ с координатами $P(\imath)=P\left\{\varepsilon_{1}=i_{1}, \ldots, \varepsilon_{m}=i_{m}\right\}, \mathbf{P}^{T} \mathbf{p}=\mathbf{p}$ и $\mathbf{P}^{n} \rightarrow \mathbf{e p}^{T}$. Определим

$$
\mathscr{Z}=\left(\mathbf{I}-\mathbf{P}+\mathbf{e p}^{T}\right)^{-1}
$$

как фундаментальную матрицу цепи $Y_{n}$. Отметим, что эта матрица уже играла важную роль в настоящей работе.

Согласно теореме 4.6.1 в [7], предельная матрица

$$
\Sigma=\lim _{n \rightarrow \infty} n^{-1} \operatorname{Cov}\left(\omega_{\imath}, \omega_{\jmath}\right)
$$

образована элементами вида

$$
\sigma_{\imath \jmath}=-\delta_{\imath \jmath} P(\imath)-P(\imath) P(\jmath)+P(\imath) \mathscr{Z}_{\imath \jmath}+P(\jmath) \mathscr{Z}_{\jmath \imath}
$$

Тем самым, если D - диагональная матрица, связанная с вектором $\mathbf{p}$, то $\Sigma$ может быть записана как

$$
\Sigma=-\mathbf{D}-(2 m-1) \mathbf{p} \mathbf{p}^{T}+\mathbf{D B}+\mathbf{B}^{T} \mathbf{D}
$$

где $\mathbf{B}=\mathscr{C}(1)=\mathscr{Z}+(m-1) \mathbf{e p}^{T}$.

Для статистических выводов необходимо иметь псевдообратную $\Sigma^{-}$ к матрице (32). Мы покажем, что

$$
\Sigma^{-}=\mathbf{D}^{-1}-\mathbf{V}
$$


где $\mathbf{V}$ определена в (12). Для начала заметим, что $\Sigma^{-} \mathbf{p}=\mathbf{e}-\mathbf{V} \mathbf{p}=0$. Согласно (14), VDB = VD + B - I, так что

$$
\mathbf{V} \Sigma=-\mathbf{I}-(2 m-1) \mathbf{e p}^{T}+\mathbf{B}+\mathbf{V B}^{T} \mathbf{D} \text {. }
$$

Имеем

$$
\mathbf{D}^{-1} \Sigma=-\mathbf{I}-(2 m-1) \mathbf{e p}^{T}+\mathbf{B}+\mathbf{D}^{-1} \mathbf{B}^{T} \mathbf{D} .
$$

Заключаем, что

$$
\left(\mathbf{D}^{-1}-\mathbf{V}\right) \Sigma=\left(D^{-1}-\mathbf{V}\right) \mathbf{B}^{T} \mathbf{D}=\left(D^{-1}-\mathbf{V}\right) \mathscr{Z}^{T} \mathbf{D}
$$

a

$$
\Sigma\left(\mathbf{D}^{-1}-\mathbf{V}\right) \Sigma=\mathbf{D} \mathscr{Z}\left(\mathbf{D}^{-1}-\mathbf{V}\right) \mathscr{Z}^{T} \mathbf{D}
$$

Для доказательства того, что матрица (33) является псевдообратной для $\Sigma$, т.е. что

$$
\Sigma \Sigma^{-} \Sigma=\Sigma
$$

остается убедиться в том, что

$$
\mathbf{D}^{-1}-\mathbf{V}=\mathscr{Z}^{-1} \mathbf{D}^{-1} \Sigma \mathbf{D}^{-1}\left(\mathscr{Z}^{T}\right)^{-1}
$$

Но матрица в правой части этого равенства есть

$$
\begin{aligned}
\mathscr{Z}^{-1} & \left(-\mathbf{D}^{-1}-\mathbf{e} \mathbf{e}^{T}+\mathscr{Z} \mathbf{D}^{-1}+\mathbf{D}^{-1} \mathscr{Z}^{T}\right)\left(\mathscr{Z}^{T}\right)^{-1} \\
= & -\mathscr{Z}^{-1} \mathbf{D}^{-1}\left(\mathscr{Z}^{T}\right)^{-1}-\mathbf{e} \mathbf{e}^{T}+\mathscr{Z}^{-1} \mathbf{D}^{-1}+\mathbf{D}^{-1}\left(\mathscr{Z}^{T}\right)^{-1} \\
= & -\left(\mathbf{I}-\mathbf{P}+\mathbf{e p}^{T}\right) \mathbf{D}^{-1}\left(\mathbf{I}-\mathbf{P}^{T}+\mathbf{p} \mathbf{e}^{T}\right)-\mathbf{e} \mathbf{e}^{T}+2 \mathbf{D}^{-1} \\
& -\mathbf{D}^{-1}\left(\mathbf{P}^{T}-\mathbf{p} \mathbf{e}^{T}\right)-\left(\mathbf{P}^{T}-\mathbf{e p}^{T}\right) \mathbf{D}^{-1} \\
= & -\left(\mathbf{P}-\mathbf{e p}^{T}\right) \mathbf{D}^{-1}\left(\mathbf{P}^{T}-\mathbf{p} \mathbf{e}^{T}\right)+\mathbf{D}^{-1}-\mathbf{e} \mathbf{e}^{T} \\
= & \mathbf{D}^{-1}-\mathbf{P} \mathbf{D}^{-1} \mathbf{P}^{T} .
\end{aligned}
$$

Формула (15) убеждает в справедливости (34).

Теорема 6.1. Для марковской иепи фиксированного порядка предельная матрица $\Sigma$ ковариаций частот $m$-грамм имеет вид (32). Если истинный порядок чепи есть $m-1$, то одна из псевдообратньх $\kappa \Sigma$ матрии есть (33).

Теорема 6.1 показывает, что ранг матрицы $\Sigma$ равен $q^{m}-q^{m-1}$. В самом деле, $\Sigma \Sigma^{-}$- идемпотентная матрица и $\operatorname{tr}\left(\Sigma \Sigma^{-}\right)=\operatorname{tr}\left(\mathbf{B}^{T}\right)-$ $\operatorname{tr}\left(\mathbf{D V B}^{T}\right)=\operatorname{tr}(\mathbf{I})-\operatorname{tr}(\mathbf{D V})=q^{m}-q^{m-1}$.

Пусть s - это $q^{m}$-мерный вектор с координатами $\sqrt{P(\imath)}, \imath \in \mathscr{I}$, так что диагональная матрица, образованная этим вектором, совпадает с $\mathbf{D}^{1 / 2}$. Положим $\Sigma_{B}=\mathbf{D}^{-1 / 2} \Sigma \mathbf{D}^{-1 / 2}$. Тогда, согласно (32),

$$
\Sigma_{B}=-\mathbf{I}-(2 m-1) \mathbf{s s}^{T}+\mathbf{D}^{1 / 2} \mathbf{B D}^{-1 / 2}+\mathbf{D}^{-1 / 2} \mathbf{B}^{T} \mathbf{D}^{1 / 2} \text {. }
$$


Ранг этой матрицы тоже есть $q^{m}-q^{m-1}$. Асимптотическим распределением нормированных частот $\left(\omega_{\imath}-n P(\imath)\right) / \sqrt{n P(\imath)}, \imath \in \mathscr{I}_{m}, m$-грамм является нормальное $N\left(0, \Sigma_{B}\right)$.

Согласно теореме 6.1, псевдообратная матрицы $\Sigma_{B}$ имеет вид

$$
\Sigma_{B}^{-}=\mathbf{D}^{1 / 2} \Sigma^{-} \mathbf{D}^{1 / 2}=\mathbf{I}-\mathbf{D}^{1 / 2} \mathbf{V} \mathbf{D}^{1 / 2} .
$$

Теорема 6.2. Пусть $\omega_{2}$ является частотой чепочки $\imath$ в $\mathrm{cma-}$ иионарной марковской чепи $\varepsilon_{1}, \ldots, \varepsilon_{n}$ порядка $m-1, P(\imath)=P\left\{\varepsilon_{1}=\right.$ $\left.i_{1}, \ldots, \varepsilon_{m}=i_{m}\right\}$. Тогда статистика

$$
\chi^{2}=\sum_{\imath \in \mathscr{I}_{m}} \frac{\left(\omega_{\imath}-n P(\imath)\right)^{2}}{n P(\imath)}-\sum_{\kappa \in \mathscr{I}_{m-1}} \frac{\left(\omega_{\kappa}-P(\kappa)\right)^{2}}{n P(\kappa)}
$$

асимптотически при $n \rightarrow \infty$ имеет $\chi^{2}$-распределение $c q^{m}-q^{m-1}$ степенями свободы.

Д ок азат ельство. Заметим, что если $Z$ - нормальный, $N\left(0, \Sigma_{B}\right)$, вектор размерности $q^{m}$, то, согласно теореме 9.2 .3 в [13], квадратичная форма $Z^{T} \Sigma_{B}^{-} Z$ имеет распределение $\chi^{2} \mathrm{c} \operatorname{tr}\left(\Sigma_{B} \Sigma_{B}^{-1}\right)=$ $q^{m}-q^{m-1}$ степенями свободы. Если обозначить через $Z_{n}$ случайный вектор с координатами $\left(\omega_{\imath}-n P(\imath)\right) / \sqrt{n P(\imath)}, \imath \in \mathscr{I}$, то $Z_{n}^{T} \Sigma_{B}^{-} Z_{n}$ совпадает с статистикой $\chi^{2}$ в $(35)$. Теорема доказана.

Частные случаи теоремы 6.2 известны для независимых случайных величин, а также в тестировании порядка марковской цепи (см., например [1]). Теорема 6.2 дает возможность проверить гипотезу, согласно которой вероятности $m$-грамм - заданные числа $P(\imath), \imath \in \mathscr{I}_{m}$. Соответствующий критерий, основанный на эмпирических частотах налагающихся цепочек, отвергнет эту гипотезу при больших значениях (35) Он обобщает так называемый сериальный тест равномерности [4].

Если при альтернативной гипотезе вероятности имеют вид $P(\imath)(1+$ $\left.n^{-1 / 2} \psi_{i}\right), \imath \in \mathscr{I}_{m}$, с $\sum P(\imath) \psi_{\imath}=0$ и $\sum \psi_{\imath} \neq 0$, то предельным распределением статистки (35) будет нецентральное $\chi^{2}$-распределение с $q^{m}-q^{m-1}$ степенями свободы и параметром нецентральности $\left(\sum \psi_{\imath}\right)^{2}$.

В этой ситуации при альтернативной гипотезе предельное распределение частот $m$-грамм $\left(\omega_{\imath}-n P(\imath)\right) / \sqrt{n P(\imath)}, \imath \in \mathscr{I}_{m}$, нормально. Вектор среднего этого распределения имеет координаты $\left(\sum \psi_{\imath}\right) \sqrt{P(\imath)}$, а матрица ковариаций есть $\Sigma_{B}$. Та же теорема 9.2 .3 из [13] показывает, что квадратичная форма $Z^{T} \Sigma_{B}^{-} Z$ имеет нецентральное $\chi^{2}$-распределение с $q^{m}-q^{m-1}$ степенями свободы и параметром нецентральности $\left(\sum \psi_{\imath}\right)^{2}$. Этот факт дает приближенную функцию мощности упомянутого критерия.

Настоящая работа была поддержана грантом MSPF-02G-068. Автор благодарен И. Бебу и рецензенту за ценные замечания. 


\section{СПИСОК ЛИТЕРАТУРЫ}

1. Billingsley P. Statistical Inference for Markov Processes. Chicago: Univ. of Chicago Press, 1961, $75 \mathrm{p}$.

2. Беляев П. Ф. О вероятности непоявления заданного числа $s$-цепочек в сложных цепях Маркова. - Теория вероятн. и ее примен., 1965, т. 10, в. 3, с. 547-551.

3. Brainerd B., Chang S.M. Number of occurrences in two-state Markov chains, with an application in linguistics. - Canad. J. Statist., 1982, v. 10, № 3, p. 225-231.

4. Good I. J. The serial test for sampling numbers and other tests for randomness. Proc. Cambridge Philos. Soc., 1953, v. 49, p. 276-284.

5. Good I. J. Quadratics in Markov-chain frequencies and the binary chain of order 2. J. Roy. Statist. Soc. Ser. B, 1963, v. 25, p. 383-391.

6. Guibas L., Odlyzko A. String overlaps, pattern matching and nontransitive games. J. Combin. Theory, 1981, v. 30, № 2, p. 183-208.

7. Кемени Дж. Дж., Снелл Дж. Л. Конечные цепи Маркова. М.: Наука, 1970, 271 с.

8. Колчин В.Ф., Севастьянов Б. А., Чистяков В.П. Случайные размещения. М.: Наука, 1976, 223 с.

9. Marsaglia G. A current view of random number generation. - Computer Science and Statistics: Proceedings of the Sixteenth Symposium on the Interface. New York: Elsevier, 1985, p. 3-10.

10. Михайлов В.Г. Асимптотическая нормальность в схеме конечно-зависимого размещения частиц по ячейкам. - Матем. сб., 1982, т. 119, № 4, с. 509-520.

11. Михайлов В.Г. Об асимптотической нормальности симметрических разделимых статистик от частот $m$-цепочек. - Дискретн. матем., 1989, т. 1, № 4, с. 92-103.

12. Odlyzko A. Asymptotic enumeration methods. - Handbook of Combinatorics. Ed. by R. L. Graham, M. Grötshel, and L. Lovácz. Amsterdam: Elsevier, 1995, p. 1063-1229.

13. Rao C.R., Mitra S.K. Generalized Inverse of Matrices and its Applications. New York: Wiley, 1971, $240 \mathrm{p}$.

14. Reinert G., Schbath S., Waterman M. G. Probabilistic and statistical properties of words: an overview. - J. Comput. Biol., 2000, v. 7, № 1-2, p. 1-46.

15. Regnier M., Szpankowski W. On pattern frequency occurrences in a Markovian sequence. - Algorithmica, 1998, v. 22, № 4, p. 631-649.

16. Rukhin A.L. Testing randomness: a suite of statistical procedures. - Теория вероятн. и ее примен., 2000 , т. 45 , в. 1, с. 137-162.

17. Rukhin A.L. Distribution of the number of words with a prescribed frequency and tests of randomness. - Adv. in Appl. Probab., 2002, v. 34, № 4, p. 775-797.

18. Соловьев А. Д. Одно комбинаторное тождество и его применение к задаче о первом наступлении редкого события. - Теория вероятн. и ее примен., 1966, т. 11, B. 2, c. 313-320.

19. Szpankowski $W$. Average Case Analysis of Algorithms on Sequences. New York: Wiley-Interscience, 2001, $551 \mathrm{p}$.

20. Тихомирова М.И., Чистяков В. П. Об асимптотике моментов числа непоявившихся $s$-цепочек. - Дискретн. матем., 1997, в. 9, № 1, с. 12-29.

21. Тихомирова М.И., Чистяков В. П. О статистических критериях отсутствующих s-грамм. - Труды по дискретной математике, т. 1. М.: ТВП, 1997, с. 265-278.

Поступила в редакцию 30.IX.2005 